\title{
Laboratory Test Original Result Unit
}

National Cancer Institute

\section{Source}

National Cancer Institute. Laboratory Test Original Result Unit. NCI Thesaurus. Code C83106.

The unit of measure for the original laboratory test result. 